\title{
Approximating Equilibrium in the Digital Marketplace
}

\author{
Jakub Konka, Craig Michie, Ivan Andonovic, and Robert Atkinson \\ Centre for Intelligent Dynamic Communications \\ University of Strathclyde \\ Glasgow G1 1XW, UK \\ Email: \{jakub.konka, c.michie, i.andonovic, robert.atkinson\}@ strath.ac.uk,
}

\begin{abstract}
Digital Marketplace is a market-based framework for trading mobile communications services at a service level. It is well suited for managing a future mobile communications environment where the one-to-one mapping between network operators and subscribers no longer holds, and the subscribers are given the option to select a network operator and a wireless access technology that matches their preferences best at a service level. As with any market-based framework, it is important to analyse the selling mechanism from the economic perspective. In this paper, we address the deficiencies of previous research on the economic equilibrium of the Digital Marketplace. We achieve this by proposing an approximation to the equilibrium of the Digital Marketplace through the use of an auction with common prior.
\end{abstract}

Keywords-Digital Marketplace; network selection; auction theory

\section{INTRODUCTION}

Mobile communications has become an indispensable part of our everyday lives. According to Ofcom [1], 51\% of all adults in the UK own a smartphone, and approximately $24 \%$ of all UK households own a tablet. Furthermore, one in five adults declares they would miss their mobile most if it were taken away. It should be noted that these numbers continue to rise, and with each year the penetration of mobile communications will increase.

Parallel to this, mobile users are given access to a plethora of wireless access technologies: from $\mathrm{WiFi}$, through $3 \mathrm{G}$, to the latest 4G. Cities throughout the UK are now offering free WiFi hotspots [2]. Furthermore, according to Ofcom [3], while 3G already covers $98 \%$ of the UK population indoors, this figure is promised to be at least matched by the $4 \mathrm{G}$ mobile services by the end of 2017 at the latest.

To make full use of this increasingly diverse environment and increase the competition between network operators even further, the one-to-one mapping between network operators and subscribers need no longer hold. This allows the subscribers to seamlessly switch not only between different wireless access technologies belonging to one particular network operator, but also between network operators themselves. In this way, the subscriber, when requesting a bearer service, is given the option to select a network operator and a wireless access technology that best matches the required quality requirements of the service: an Always Best Connected paradigm [4]. It is not only to the benefit of the subscribers, however, since the integration of wireless access technologies will allow network operators for more efficient usage of network resources.

In order to manage such a complex system, a sophisticated management platform is required. In this research, we advocate the use of the Digital Marketplace (DMP). DMP is a market-based framework where network operators compete in a procurement auction for the right to transport the subscriber's requested service over their infrastructure [5], [6]. As with any market-based framework, it is important to analyse the selling mechanism from the economic perspective. In [6]-[8], Konka et al. present the results of such an analysis. They characterise the equilibrium, and propose numerical methods for the numerical approximation of the equilibrium, which is otherwise analytically intractable. The proposed numerical methods are, however, deficient in the sense that they apply only to a subset of all bidding scenarios possible within the DMP. In this paper, we address this problem by modelling the selling mechanism in the DMP as an auction with common prior. In an auction with common prior, the range the costs can vary is the same for each bidder, and for this type of auctions, there exists a plethora of well-studied numerical algorithms that can be used to approximate the equilibrium of the auction. Consequently, we propose an approximation to the equilibrium of the DMP through the use of an auction with common prior [9]. Our solution yields results of acceptable quality, with the approximation error never exceeding 16\%, and importantly, decreasing with the increasing number of network operators.

The rest of this paper is organized as follows. In Section II, a brief overview of the selling mechanism in the DMP is given. Section III explains the concept of auction with common prior, while Section IV describes how the DMP selling mechanism can be approximated as an auction with common prior. In Section V, a methodology for quantifying how well the common prior auction models the DMP auction is given, while Section VI provides numerical results of approximations in several scenarios. Finally, Section VII draws conclusions.

\section{Bidding Problem in the Digital Marketplace}

In order to put the research work presented in this paper into context, the bidding problem in the DMP is briefly summarised here. The DMP features an auction-based network 
selection mechanism, which is based on a procurement firstprice sealed-bid auction. Following the notation of Konka et al. [6], there are $n=|N|$ network operators (NOs) who bid for the right to sell their product to the subscriber. Each NO is characterised by utility function

$$
u_{i}(\hat{b}, \hat{c})= \begin{cases}\frac{1}{w}\left(\hat{b}_{i}-\hat{c}_{i}\right) & \text { if } \hat{b}_{i}<\min _{j \neq i} \hat{b}_{j}, \\ 0 & \text { if } \hat{b}_{i}>\min _{j \neq i} \hat{b}_{j} .\end{cases}
$$

where $\hat{b}=\left(\hat{b}_{i}, \hat{b}_{-i}\right)$ is an $n$-tuple of all NOs bids, $\hat{c}=\left(\hat{c}_{i}, \hat{c}_{-i}\right)$ is an $n$-tuple of all NOs costs, and $w \in[0,1]$ denotes the weight that the subscriber attaches to the price of the service, which is inversely proportional to the weight attached to the reputation of the particular NO by the subscriber. Furthermore, the costs for each $\mathrm{NO}$ are distributed over the interval

$$
\hat{c}_{i} \in\left[\underline{\hat{c}}_{i}, \overline{\hat{c}}_{i}\right] \equiv\left[(1-w) r_{i},(1-w) r_{i}+w\right],
$$

where $r_{i} \in[0,1]$ denotes the reputation for each NO.

As shown in [6], there exists a unique equlibrium to this bidding problem, and it is characterised by the following system of ordinary differential equations (ODEs)

$$
\frac{d}{d b} \hat{c}_{i}(b)=\frac{1-F_{i}\left(\hat{c}_{i}(b)\right)}{f_{i}\left(\hat{c}_{i}(b)\right)}\left[\frac{1}{n-1} \sum_{k=1}^{n} \frac{1}{b-\hat{c}_{k}(b)}-\frac{1}{b-\hat{c}_{i}(b)}\right]
$$

for $i=1,2, \ldots, n$, with the upper boundary condition

$$
\hat{c}_{i}(\overline{\hat{b}})=\overline{\hat{b}},
$$

and the lower boundary condition

$$
\hat{c}_{i}(\underline{\hat{b}})=\min \left\{\underline{\hat{c}}_{i}, \hat{c}(\underline{\hat{b}})\right\}
$$

for all $i \in N$. Here, $\hat{c}_{i}$ denotes the inverse bidding functions for all NOs $i \in N$, while $F_{i}$ denotes the distribution function of $\hat{c}_{i}$. It is possible to derive the analytical solution to the system in Eq. (2) in the case of $n=2$ bidders and $F_{i}$ corresponding to uniform distribution. However, for more than 2 bidders, a closed-form solution does not exist.

Two numerical algorithms for approximating the solution to the system in Eq. (2) are proposed in [6]: forward shooting method (FSM), and polynomial projection method (PPM). Both methods were first proposed by Bajari [10] for approximating auctions with common prior, and were adapted to the DMP bidding problem in [6]. To give a brief overview of each method, the FSM is an adaptation of a finite differences method for approximating solutions to ODEs, while PPM tries to find the best fitting polynomials to the equilibrium bidding functions. Due to the nature of the problem, however, the algorithms handle only a subset of all cases such that the lower boundary condition (4) is reduced to $\hat{c}_{i}(\underline{\hat{b}})=\underline{\hat{c}}_{i}$. It is the purpose of this paper to address this deficiency by casting the bidding problem into a simpler setting of common prior (CP) for which there exists a plethora of numerical solutions that are well-researched and well-defined [9].

\section{AUCTION WITH COMMON PRIOR}

In an auction with $\mathrm{CP}$, we assume that each $\mathrm{NO} i$ draws their cost from common support across all bidders; that is, each NO draws their cost from the interval

$$
\hat{c}_{i} \in[\underline{\hat{c}}, \overline{\hat{c}}] \quad \text { for all } i \in N .
$$

In this case, the equilibrium is still characterised by the system of ODEs in Eq. (2), however, with different boundary conditions; that is, with the following upper boundary condition

$$
\hat{c}_{i}(\overline{\hat{b}})=\overline{\hat{c}},
$$

and the lower boundary condition

$$
\hat{c}_{i}(\underline{\hat{b}})=\underline{\hat{c}}
$$

for all $1 \leq i \leq n$.

As shown by Lebrun [11], assuming the assumptions governing the original DMP bidding problem described in [6] hold, there exists a unique solution to the system of ODEs (2). Furthermore, similarly to the original bidding problem, the closed-form solution exists only in a limited number of special cases [9], [12]. However, as presented by Hubbard and Paarsch [9], there exist well-defined numerical methods which can be used to solve it.

In particular, in this paper, the $\mathrm{CP}$ auction is approximated using the FSM method. It was chosen due to its relatively low implementation complexity (compared to the PPM method), and the fact that its variant is also used to approximate the DMP bidding problem. Therefore, in terms of the numerical accuracy and stability, the numerical solutions to the DMP and $\mathrm{CP}$ auctions should be of comparable quality.

\section{Digital Marketplace Cast into COMMON PRior SETTING}

In a $\mathrm{CP}$ auction, every $\mathrm{NO}$ is characterised by a distribution (of costs) with common support across all NOs. Hence, in order to model the DMP auction as an auction with CP, firstly, we need to agree on a support that is common to every NO and, at the same time, encompasses the supports of every individual NO from the original DMP auction. The smallest such support is

$$
[\underline{\hat{c}}, \overline{\hat{c}}]=\left[\min _{i \in N}\left\{\hat{\underline{c}}_{i}\right\}, \max _{i \in N}\left\{\overline{\hat{c}}_{i}\right\}\right] \subset[0,1] .
$$

To see this, recall that, for any given $w \in(0,1)$, assuming $r_{1} \leq \cdots \leq r_{n}$ with at least one inequality strict, it follows $\underline{\hat{c}}_{1} \leq \cdots \leq \underline{\hat{c}}_{n}$ and $\overline{\hat{c}}_{1} \leq \cdots \leq \overline{\hat{c}}_{n}$ with at least one inequality strict. If we further let $C_{i}=\left[\underline{\hat{c}}_{i}, \overline{\hat{c}}_{i}\right]$ then $C=\bigcup_{i \in N} C_{i}$ is the smallest set containing all sets $C_{i}$ for all $i \in N$. Since $C_{i}$ is closed for all $i \in N$, it follows that $C$ is closed, and $C=[\underline{\hat{c}}, \overline{\hat{c}}]$ such that $\underline{\hat{c}} \leq \underline{\hat{c}}_{i}$ and $\overline{\hat{c}}_{i} \leq \overline{\hat{c}}$ for all $i \in N$, which is equivalent to $\left[\min _{i \in N}\left\{\underline{\hat{c}}_{i}\right\}, \max _{i \in N}\left\{\overline{\hat{c}}_{i}\right\}\right]$.

All that remains is to then select a family of distributions which captures the numerical ranges of the original supports as closely as possible. To provide an illustrative example, let there be 2 NOs such that $\underline{\hat{c}}_{1}<\underline{\hat{c}}_{2}<\overline{\hat{c}}_{1}<\overline{\hat{c}}_{2}$. Each NO is characterised by a uniform distribution. One possible way 
TABLE I

BIDDING SCENARIO WITH 2 NETWORK OPERATORS

\begin{tabular}{|lcc|}
\hline & Price weight, $w$ & Reputation rating, $r_{i}$ \\
\hline Network operator 1 & \multirow{2}{*}{0.5} & 0.25 \\
Network operator 2 & & 0.75 \\
\hline
\end{tabular}

of casting this scenario into common prior setting is to model the distributions of both NOs as truncated normal distributions truncated to the interval $\left[\underline{\hat{c}}_{1}, \overline{\hat{c}}_{2}\right]$, and with differing mean and standard deviation parameters.

In order to describe the truncated normal distribution, firstly recall the probability density function (pdf) of standard normal distribution

$$
\phi(\hat{c})=\frac{1}{\sqrt{2 \pi}} \exp \left\{-\frac{1}{2} \hat{c}^{2}\right\},
$$

and cumulative distribution function (cdf)

$$
\Phi(\hat{c})=\int_{-\infty}^{\hat{c}} \phi(\hat{c}) d \hat{c}=\frac{1}{2}\left[1+\operatorname{erf}\left(\frac{\hat{c}}{\sqrt{2}}\right)\right]
$$

for all $\hat{c} \in \mathbb{R}$. The pdf of the truncated normal distribution, truncated to the interval $\hat{c} \in[\underline{\hat{c}}, \overline{\hat{c}}]$, can then be described in terms of the pdf of the standard normal distribution as follows

$$
f(\hat{c} ; \mu, \sigma, \hat{\hat{c}}, \overline{\hat{c}})=\frac{\frac{1}{\sigma} \phi\left(\frac{\hat{c}-\mu}{\sigma}\right)}{\Phi\left(\frac{\overline{\hat{c}}-\mu}{\sigma}\right)-\Phi\left(\frac{\hat{\underline{c}}-\mu}{\sigma}\right)}
$$

where $\mu \in \mathbb{R}$ is the mean (or location) of the distribution, and $\sigma^{2} \geq 0$ is the variance (or squared scale) [13]. Similarly, the cdf of the truncated normal distribution can be defined as follows

$$
F(\hat{c} ; \mu, \sigma, \underline{\hat{c}}, \overline{\hat{c}})=\frac{\Phi\left(\frac{\hat{\hat{c}}-\mu}{\sigma}\right)-\Phi\left(\frac{\hat{\hat{c}}-\mu}{\sigma}\right)}{\Phi\left(\frac{\overline{\hat{c}}-\mu}{\sigma}\right)-\Phi\left(\frac{\hat{\hat{c}}-\mu}{\sigma}\right)} .
$$

By way of example, consider bidding scenario summarized in Table I. Suppose we were to cast this scenario into common prior setting where NOs are characterised by truncated normal distributions. Firstly, we note that the supports for both NOs are

$$
\left[\underline{\hat{c}}_{1}, \overline{\hat{c}}_{1}\right]=[0.125,0.625]
$$

for NO 1, and

$$
\left[\underline{\hat{c}}_{2}, \overline{\hat{c}}_{2}\right]=[0.375,0.875]
$$

for NO 2, while the common support is given by

$$
[\underline{\hat{c}}, \overline{\hat{c}}]=\left[\underline{\hat{c}}_{1}, \overline{\hat{c}}_{2}\right]=[0.125,0.875] .
$$

Secondly, we need to specify distribution specific parameters (mean and standard deviation) for each NO. Since the aim is to approximate the original distributions of both NOs, we pick the midpoints of the original supports as means, that is,

$$
\mu_{i}=\underline{\hat{c}}_{i}+\frac{\overline{\hat{c}}_{i}-\hat{\hat{c}}_{i}}{2}=\underline{\hat{c}}_{i}+\frac{w}{2},
$$

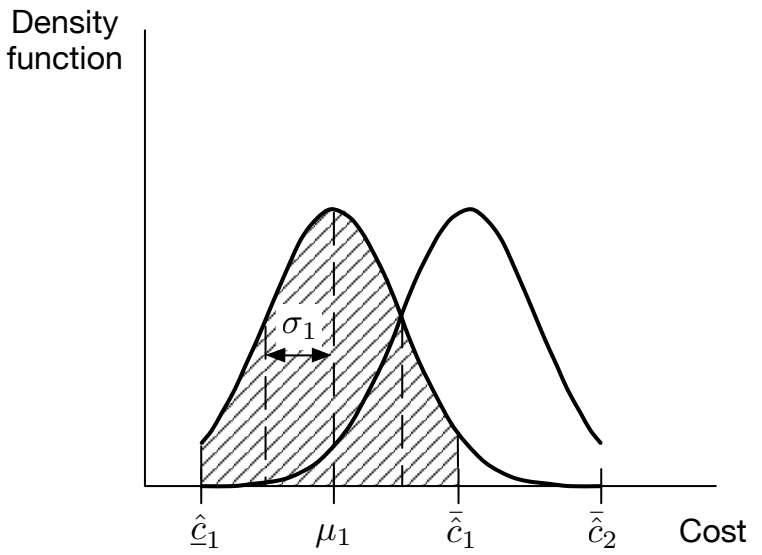

Fig. 1. Choosing parameters for the truncated normal distributions of NOs

TABLE II

NUMERICAL VALUES OF THE CHOSEN TRUNCATED NORMAL DISTRIBUTION PARAMETERS

\begin{tabular}{|ccc|}
\hline & Mean, $\mu_{i}$ & Standard deviation, $\sigma_{i}$ \\
\hline Bidder 1 & 0.375 & 0.125 \\
Bidder 2 & 0.625 & 0.125 \\
\hline
\end{tabular}

and let the standard deviations be equal to the quarter of the length of the original supports, that is,

$$
\sigma_{i}=\frac{\overline{\hat{c}}_{i}-\hat{\hat{c}}_{i}}{4}=\frac{w}{4} .
$$

The choice of the parameters is motivated by the shape of the normal distribution, and the fact that, with this choice of parameters, the probability of at least 0.95 of drawing cost from the interval $\left[\underline{\hat{c}}_{i}, \overline{\hat{c}}_{i}\right]$ (which corresponds to the interval $\left.\left[\mu_{i}-2 \sigma_{i}, \mu_{i}+2 \sigma_{i}\right]\right)$ is achieved [13]; therefore, minimising the probability of drawing cost from outside the interval $\left[\hat{\hat{c}}_{i}, \overline{\hat{c}}_{i}\right]$, and effectively imitating uniform distribution with support $\left[\underline{\hat{c}}_{i}, \overline{\hat{c}}_{i}\right]$. This is depicted in Fig. 1 as the shaded region under the bell curve, while Table II summarizes the numerical values of the described parameters.

\section{Methodology for QuANTIFying ACCURACy OF THE APPROXIMATIONS}

In order to quantify how well the $\mathrm{CP}$ auction models the original DMP auction, we shall consider two metrics: subscriber's expected price, and ex ante expected utility for each NO. In this way, we obtain an indicator of how better off (or worse off) is the subscriber and each of the NOs.

The subscriber's expected price is equivalent to the expected value of the winning bid; that is,

$$
p=E\left[\hat{b}_{i}\left(\hat{c}_{i}\right) \mid \hat{b}_{i}\left(\hat{c}_{i}\right)<\min _{j \neq i} \hat{b}_{j}\left(\hat{c}_{j}\right)\right],
$$

where $\hat{b}_{i}$ is the equilibrium bidding function for all $i \in N$. Since an analytical derivation of the closed-form solution is not straightforward, we resort to numerical estimation of the buyer's expected price. That is, for each considered bidding 
scenario, the costs for each $\mathrm{NO}$ are pseudo-randomly drawn from uniform distribution, the corresponding equilibrium bids are computed, and the minimum is chosen as the winning bid (price). This procedure is repeated 1000 times, yielding 1000 i.i.d. observations of the price which are then averaged to give an estimate of the expected price (consequence of the Strong Law of Large Numbers [14]).

In order to define the NO's ex ante expected utility, we define the expected utility function for each NO $i \in N$ as

$$
\Pi_{i}\left(\hat{c}_{i}\right)=\left(\hat{b}_{i}\left(\hat{c}_{i}\right)-\hat{c}_{i}\right) \cdot \prod_{j \neq i}\left(1-F_{j}\left(\hat{b}_{j}^{-1}\left(\hat{b}_{i}\left(\hat{c}_{i}\right)\right)\right)\right)
$$

where $\hat{b}_{i}$ is the equilibrium bidding function, and $F_{i}$ is the distribution function of costs for $\mathrm{NO} i$. The ex ante expected utility is then equivalent to the expected value of the expected utility; that is,

$$
\Pi_{i}=E\left[\Pi_{i}\left(\hat{c}_{i}\right)\right]=\int_{\underline{\underline{c}}_{i}}^{\overline{\hat{c}}_{i}} \Pi_{i}(t) d F_{i}(t)
$$

for all $i \in N$. In other words, the ex ante expected utility can be thought of as the average expected utility for each NO for each considered bidding scenario, and it follows from the definition of ex ante expected payments in a standard firstprice auction put forward by Krishna [12].

The way the aforementioned metrics are actually computed deserves a more elaborate explanation. The numerical derivation of equilibrium in the $\mathrm{CP}$ auction relies on approximating the NOs' distributions of costs with truncated normal distributions with common support. When computing the expected price and ex ante expected utilities for all NOs in the $\mathrm{CP}$ auction, it is assumed, however, that the NOs draw their costs from their actual (uniform) distributions but use the equilibrium bidding strategies derived for the $\mathrm{CP}$ auction with truncated normal distributions to compute their bids. In this way, when computing the expected price and ex ante expected utilities, we do not misrepresent the NOs' distributions of costs, and hence, ensure the comparison results of casting the DMP auction into CP auction setting are as realistic as possible.

We further define the relative error in expected prices as

$$
\eta_{p}=\left|\frac{p^{D M P}-p^{C P}}{p^{D M P}}\right|
$$

and the relative error in ex ante expected utilities as

$$
\eta_{\Pi_{i}}=\left|\frac{\Pi_{i}^{D M P}-\Pi_{i}^{C P}}{\Pi_{i}^{D M P}}\right|
$$

for all $i \in N$, where $p^{D M P}$ and $p^{C P}$ denote the expected prices for DMP and CP auction respectively, and $\Pi_{i}^{D M P}$ and $\Pi_{i}^{C P}$ denote the ex ante expected utilities for NO $i$ for DMP and $\mathrm{CP}$ auction respectively.

\section{APPROXIMATION RESULTS}

We analyse the results for three bidding scenarios: with $n=2, n=3$ and $n=4$ NOs respectively. We concentrate on only upto 4 NOs since with each additional NO, the time required to simulate the problem increases exponentially. Furthermore, since the UK market is currently dominated by an oligopoly of four NOs who own their infrastucture (EE, Vodafone, $\mathrm{O} 2$ and Three), solving the bidding problem for 4 NOs is directly relevant. The procedure for generating the approximation results is as follows:

1) For each chosen value of price weight, generate 100 reputation ratings $n$-tuples, $\left(r_{1}, \ldots, r_{n}\right)$. Each $n$-tuple is ordered; that is, $r_{1}<r_{2}<\cdots<r_{n}$. Therefore, in what follows, NO 1 is characterised by the lowest reputation rating, NO 2 by the second lowest, and so on. By ordering individual reputation ratings within the $n$-tuples, we focus on exploring the mean relative errors in ex ante expected utilities for individual NOs characterised by the lowest reputation rating, second lowest, etc. In other words, if a NO is characterised by the lowest reputation rating, we quantify the mean relative error in ex ante expected utility the $\mathrm{NO}$ is going to incur by bidding according to the equilibrium bidding strategies prescribed by the $\mathrm{CP}$ auction. Without this assumption, the mean relative error curves would converge on the same value for all NOs, and thus, some valuable insight into the extent of the mean relative errors in ex ante expected utilities would be lost. It is worth noting, however, that the mean relative error in expected price is unaffected by ordering of the reputation ratings.

Furthermore, each $r_{i}$ for each NO $i$ is drawn from a uniform distribution over the range $(0,1)$. In order to keep the analysis numerically tractable, we do not consider bidding scenarios with NOs characterised by equal reputation ratings.

2) For each reputation ratings $n$-tuple, evaluate relative errors in expected price and ex ante expected utility per NO using Eqs. (15) and (16).

3) Evaluate mean relative errors in expected price and ex ante expected utility per NO, and associated $95 \%$ confidence intervals.

4) Repeat for price weight values ranging from 0.75 to 0.99 . Price weight values are bounded from below by 0.75 which is in line with the work presented in [6], and it guarantees that the lower boundary condition in Eq. (4) reduces to $\hat{c}_{i}(\underline{\hat{b}})=\underline{\hat{c}}_{i}$. Otherwise, as reported in [6], it would be impossible to numerically approximate solutions to the DMP auction using the FSM and PPM methods as they are not defined for the problem with unreduced lower boundary condition in Eq. (4).

\section{A. $n=2$ Network Operators}

The approximation results for two NOs are depicted in Fig. 2. It is worth observing that as the price weight increases, the confidence intervals for the mean relative errors decrease. This is a direct consequence of the fact that as the price weight $w \rightarrow 1$, the actual values of the reputation ratings of the NOs do not significantly influence the mean relative 


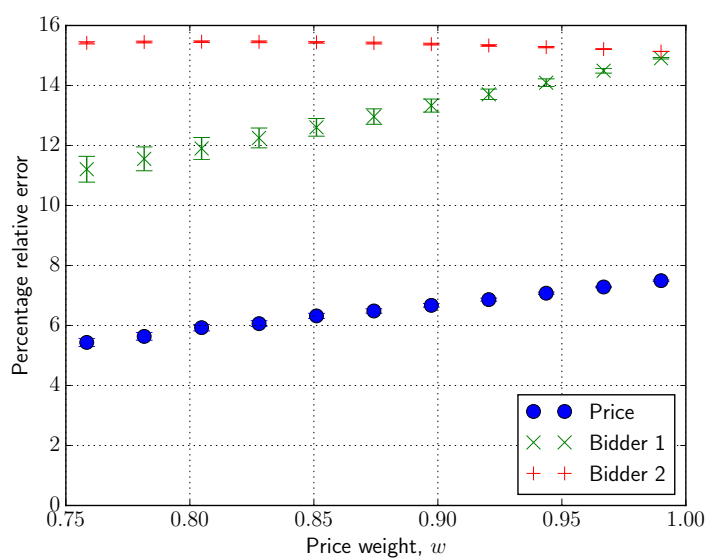

Fig. 2. Approximation results for 2 NOs

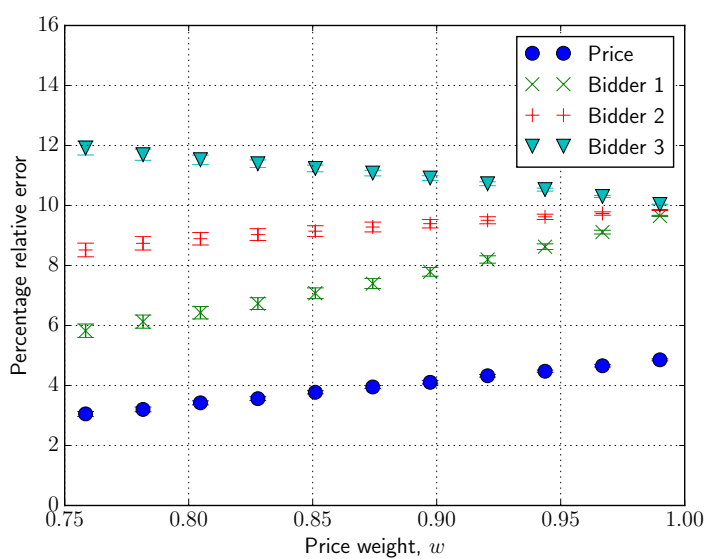

Fig. 3. Approximation results for 3 NOs errors in expected price and ex ante expected utilities for both NOs. To see this, recall from Eq. (7) the common support $\left[\min _{i} \underline{c}_{i}, \max _{i} \bar{c}_{i}\right]=\left[(1-w) \min _{i} r_{i},(1-w) \max _{i} r_{i}+w\right]$. As $w \rightarrow 1$, this reduces to $\left[\lim _{w \rightarrow 1}(1-w) \min _{i} r_{i}, \lim _{w \rightarrow 1}(1-\right.$ $\left.w) \max _{i} r_{i}+w\right]=[0,1]$. Hence, as the price weight increases, the less significant the effect of the reputation ratings on the common support.

Furthermore, as the price weight approaches 1, the mean relative errors in ex ante expected utilities for both NOs start to converge. This is due to the fact that, as $w \rightarrow 1$ and in particular at $w=1$, the DMP auction becomes a standard first-price auction with all NOs characterised by uniform distributions which are overlapping to a high degree; i.e., with some abuse of notation, $F_{i}(x) \approx F_{j}(x)$ for all $x$, $i \neq j$ and $i, j \in N$. The same is true for the CP auction with this difference that all NOs are characterised by almost equal truncated normal distributions. Furthermore, in both auctions, the NOs are characterised by symmetric, albeit different across auctions, equilibrium bidding strategies. This is due to the fact that at a symmetric equilibrium the support becomes identical in both auctions, and hence, uniform distribution of costs and truncated normal distribution of costs have to result in different equilibrium bidding strategies. This in turn leads to almost equal mean relative errors in ex ante expected utilities for all NOs.

The mean error in expected prices is approximately linearly increasing in price weight, and is bounded from above by $8 \%$ and from below by $4 \%$. The mean error in ex ante expected utility for NO 1 also linearly increasing in price weight, and is bounded from above by $15 \%$ and from below by $10 \%$. For NO 2 , however, the relationship between the price weight and the mean error is nonlinear, with the error attaining its maximum of approximately $15.5 \%$ for the price weight of $w \approx 0.8$. It is bounded from above by $15.5 \%$ and from below by $14 \%$. It is clear that NO 1 who is characterised by lower reputation rating is experiencing overall smaller mean error for all values of the price weight. However, as $w \rightarrow 1$ and as explained in the previous paragraph, the mean error converges on the same value of approximately $15 \%$ for both NOs.

\section{B. $n=3$ Network Operators}

Figure 3 depicts the approximation results for three NOs. First of all, it should be noted that the first two observations pointed out in case of two NOs also apply to the current case of three NOs. All mean relative errors, unlike in the case of two NOs, however, exhibit clear nonlinearity in price weight. Furthermore, the mean relative error in expected prices is increasing as the price weight increases, and achieves its maximum at $w=0.99$. It is bounded from above by $5 \%$ and from below by $2 \%$. The mean relative error in ex ante expected utilities for NO 1 is bounded from above by $10 \%$ and from below by $5 \%$. The mean relative error in ex ante expected utilities for NO 2 is also bounded from above by $10 \%$, but it is bounded from below by $8 \%$. It is worth noting that the shape of the mean relative error curve for NO 2 resembles that of the mean relative error curve for NO 1 translated in ydirection. Finally, the mean relative error in ex ante expected utilities for NO 3 is bounded from above by approximately $12 \%$ and from below by $10 \%$.

As expected, NO 3 who is characterised by the highest reputation rating experiences the highest mean relative error in $e x$ ante expected utilities for all values of the price weight out of all NOs. In fact, the lower bound for NO 3 is the same as the upper bound for the remaining NOs. This agrees with the conclusion drawn for the case of two NOs, where NO 2 was the NO characterised by the highest reputation rating and experienced the highest mean relative error out of all NOs.

\section{C. $n=4$ Network Operators}

Figure 4 depicts the approximation results for four NOs. Firstly, it should be noted that, similarly to the previous 


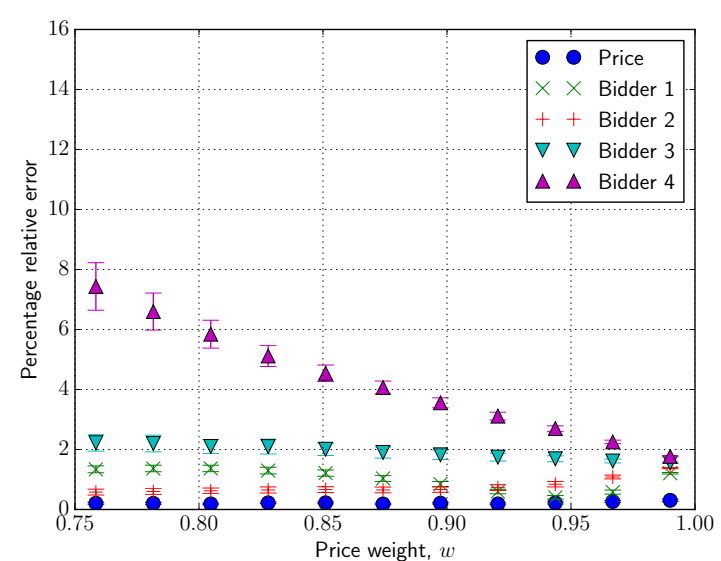

Fig. 4. Approximation results for 4 NOs

two scenarios, as the price weight approaches 1 , the mean relative errors in ex ante expected utilities for all NOs start to converge. Furthermore, as the price weight increases, the confidence intervals for the mean relative errors decrease. In terms of shape of the mean relative error curves, similarly to the case of three NOs, all mean relative errors exhibit some nonlinearity in price weight. Furthermore, the mean relative error in expected prices is bounded from above by $1 \%$, and from below by approximately $0.1 \%$. The mean relative error in ex ante expected utilities for NO 1 is bounded from above by $2 \%$, and from below by approximately $0.1 \%$. The mean relative error in ex ante expected utilities for NO 2 is bounded from above by $2 \%$, and from below by $0.1 \%$. It is worth noting that, for the values of price weight $w \in[0.75,0.9]$, the mean relative error for NO 2 is actually smaller than for NO 1, even though NO 1 is characterised by the lowest reputation rating. The mean relative error in ex ante expected utilities for NO 3 is bounded from above by $3 \%$, and from below by $1.5 \%$. Finally, the mean relative error for NO 4 is bounded from above by $8 \%$, and from below by $1.5 \%$.

As expected, NO 4 who is characterised by the highest reputation rating experiences the highest mean relative error in ex ante expected utilities for all values of the price weight out of all NOs. This agrees with the conclusion drawn for the previous two bidding scenarios, where NO who was characterised by the highest reputation rating, experienced the highest mean relative error out of all NOs.

\section{Discussion}

Considering all bidding scenarios together, it should be noted that, for all considered price weight values, the mean relative error in expected prices is decreasing as the number of NOs increases. Similarly, the mean relative error in ex ante expected utilities for all NOs decreases as the number of NOs increases. As a consequence, as the number of NOs increases, the mean relative error for the price weight approaching 1 decreases as well. However, for NO characterised by the highest reputation rating, we observe that, as the number of
NOs increases, the range of mean relative errors grows larger. All in all, it can be concluded that the CP auction becomes a more accurate approximation to the DMP auction with the increasing number of NOs.

\section{CONCLUSIONS}

In this paper, we have shown how a selling mechanim employed by the Digital Marketplace can be modelled as an auction with common prior. For this type of auctions, there exists a plethora of well-studied numerical algorithms that can be used to approximate the equilibrium of the auction.

The paper has explored 3 bidding scenarios: with $n=2$, $n=3$ and $n=4$ network operators. It was shown that the network operator characterised by the highest reputation rating will incur the highest approximation error in all cases. Furthermore, it was also shown that, as the number of network operators increases, the approximation error decreases.

All in all, it can be concluded that approximating the selling mechanism employed by the Digital Marketplace with a common prior auction constitutes a valid alternative, and as such, even though not perfectly accurate, it might be a more desirable option for the network operators due to the wealth of numerical methods available that have been extensively studied by the researchers.

\section{REFERENCES}

[1] Ofcom, "Communications Market Report 2013." [Online]. Available: http://stakeholders.ofcom.org.uk/binaries/research/cmr/cmr13/2013 UK_CMR.pdf [Available Oct. 12, 2014].

[2] $\mathrm{BBC}$, "Free Wifi internet access spots now live across Glasgow." [Online]. Available: http://www.bbc.co.uk/news/ uk-scotland-glasgow-west-28247667 [Accessed Oct. 12, 2014].

[3] Ofcom, "Ofcom announces winners of the 4G mobile auction." [Online]. Available: http://media.ofcom.org.uk/news/2013/ winners-of-the-4g-mobile-auction/ [Accessed Oct. 12, 2014].

[4] E. Gustafsson and A. Jonsson, "Always Best Connected," Wireless Communications, IEEE, vol. 10, no. 1, pp. 49-55, 2003.

[5] G. Le Bodic, D. Girma, J. Irvine, and J. Dunlop, "Dynamic 3G Network Selection for Increasing the Competition in the Mobile Communications Market," in Vehicular Technology Conference, 2000. IEEE VTS-Fall VTC 2000. 52nd, vol. 3, pp. 1064-1071, 2000.

[6] J. Konka, C. Michie, I. Andonovic, and R. Atkinson, "Auction-based Network Selection in a Market-based Framework for Trading Wireless Communications Services,"Vehicular Technology. IEEE Transactions on, vol. 63, pp. 1365-1377, March 2014.

[7] J. Konka, R. Atkinson, and J. Irvine, "Economic Aspects of Intelligent Network Selection: A Game-Theoretic Approach," in Mobile Ubiquitous Computing, Systems, Services and Technologies, 2011. UBICOMM '11. The Fifth International Conference on, 20-25 Nov. 2011.

[8] J. Konka, J. Irvine, and R. Atkinson, "Economics of Intelligent Selection of Wireless Access Networks in a Market-based Framework: A GameTheoretic Approach," International Journal on Advances in Networks and Services, vol. 5, no. 3 \& 4, pp. 210-224, 2012.

[9] T. P. Hubbard and H. J. Paarsch, "On the Numerical Solution of Equilibria in Auction Models with Asymmetries within the Private-Value Paradigm," Working Paper, Texas Tech University, Nov. 2011.

[10] P. Bajari, "Comparing competition and collusion: a numerical approach," Economic Theory, vol. 18, pp. 187-205, July 2001.

[11] B. Lebrun, "Uniqueness of the equilibrium in first-price auctions," Games and Economic Behavior, vol. 55, no. 1, pp. 131-151, 2006.

[12] V. Krishna, Auction Theory. Academic Press, second ed., 2010.

[13] N. L. Johnson, S. Kotz, and N. Balakrishnan, Continuous Univariate Distributions, vol. 1 of Wiley Series in Probability and Mathematical Statistics, ch. 13, pp. 80-206. John Wiley \& Sons, second ed., 1994.

[14] A. M. Law, Simulation Modeling \& Analysis, ch. 4, pp. 214-242. McGraw-Hill Internation Edition, fourth ed., 2007. 